VARIA 


\title{
La faiblesse des partis agraires en France : le cas du «parti de l'agriculture» sous le Second Empire
}

Chloé Gaboriaux ${ }^{1}$

\begin{abstract}
Résumé - Peu connus des chercheurs, les débats soulevés à la fin des années 1860 par les vains efforts d'un publiciste pour lancer un « parti de l'agriculture » sont pourtant riches d'enseignements. À un moment où les partis engagent un effort de structuration qui débouche, à la fin du XIXe siècle, sur la constitution de partis au sens moderne du terme, l'échec du " parti de l'agriculture » permet de mieux comprendre le paradoxe que constitue l'absence d'un parti agrarien ou paysan dans une France où les ruraux ont longtemps représenté une écrasante majorité électorale. La méfiance à l'égard des partis, souvent invoquée, ne suffit pas en effet à rendre compte de l'échec du parti de l'agriculture sous le Second Empire. Elle constitue surtout un argument efficace pour ses adversaires, dans un conflit que les électeurs ruraux finissent par trancher aux dépens de leurs porte-parole autoproclamés : réticente à confondre ses intérêts avec ceux des élites agricoles, la paysannerie s'est aussi montrée sensible aux discours de leurs adversaires, qui n'ont pas hésité à les dénoncer comme d'anciens notables soucieux de rétablir les relations féodales. Dans une France où les rapports de force sont puissamment déterminés par l'imaginaire social et politique légué par la Révolution, le " parti de l'agriculture " avait peu de chances de réussite, non pas en dépit mais en raison même de la prépondérance électorale des ruraux.
\end{abstract}

Mots clés - partis politiques, monde rural, agrarisme, représentation, France du XIXe siècle

1. Je remercie vivement les évaluateurs de cet article pour leurs remarques et suggestions très pertinentes. 
Il est extraordinaire qu'il n'y ait jamais eu un véritable parti paysan dans 《 ce pays où le secteur primaire représente à l'heure actuelle $35,5 \%$ », écrivait Georges Lavau en $1953^{2}$. La remarque pourrait être discutée au regard de l'expérience des Comités de défense paysanne et du Parti agraire et paysan français de l'entre-deux-guerres ou, plus proche du moment où écrit Georges Lavau, de celle du Parti paysan d'après-guerre, intégré alors au Centre national des indépendants et paysans. Mais ces expériences partisanes sont en effet loin d'avoir l'ampleur de celles qu'ont connues les pays scandinaves ou certains pays d'Europe centrale et orientale ${ }^{3}$.

Les chercheurs qui se sont penchés sur la question sont peu nombreux. On ne s'en étonnera pas: les partis agraires suscitant en général peu d'intérêt ${ }^{4}$, les moins puissants d'entre eux sont a fortiori négligés. Deux hypothèses ont notamment été formulées pour rendre compte de la faiblesse des partis agraires en France. La première relève de la culture politique : l'universalisme français aurait nourri une méfiance durable à l'égard des partis, longtemps synonymes de division et d'agitation, en particulier lorsqu'ils prétendaient s'appuyer sur un groupe social. C'est celle de Georges Lavau qui y voit la raison pour laquelle ils se seraient structurés si tardivement et tiendraient à se présenter chacun " comme une abstraction épurée de toute relation compromettante avec une classe sociale, une catégorie économique ou une croyance religieuse ${ }^{5} »$.

La seconde est avancée par Seymour M. Lipset et Stein Rokkan, dont le modèle des clivages est multifactoriel. Ils affirment que les partis agraires émergent dans les pays qui, comme la France, sont encore ruraux au moment de l'élargissement du droit de vote, accueillent une paysannerie largement indépendante des élites rurales et présentent une frontière culturelle entre les villes et les campagnes, mais qui, contrairement à la France, donnent à l'Église catholique une influence peu significative. L'absence de parti agraire s'expliquerait donc en général par l'importance de l'Église catholique, qui empêcherait l'autonomisation politique des intérêts agrariens au profit d'un front conservateur puissant, associant la défense de la religion à celle de la civilisation rurale et

2. Lavau (G.), Partis politiques et réalités sociales : contribution à une étude réaliste des partis politiques, Paris, Armand Colin, 1953, p. 141.

3. De Waele (J.-M.), Seiler (D.-L.) dir., Les partis agrariens et paysans en Europe, Bruxelles, Éditions de l'Université de Bruxelles, 2009.

4. Daniel-Louis Seiler et Jean-Michel De Waele parlent même de «myopie des politistes à leur encontre » dans leur introduction à l'ouvrage précédemment cité : «La défense du monde rural : les partis agrariens et paysans ", ibid., p. 8.

5. Lavau (G.), Partis politiques et réalités sociales..., op. cit., p. 139. Cf. aussi Huard (R.), La naissance du parti politique en France, Paris, Presses de Sciences Po, 1996 ; Rosanvallon (P.), Le peuple introuvable : histoire de la représentation démocratique en France, Paris, Gallimard, 1998, p. 225 et s. ; Fretel (J.), Lefebvre (R.), « La faiblesse des partis politiques français : retour sur un lieu commun historiographique», in Offerlé (M.), Rousso (H.), dir., La fabrique interdisciplinaire : histoire et science politique, Rennes, Presses universitaires de Rennes, 2008. 
du traditionalisme (explicitement monarchique au XIX ${ }^{\mathrm{e}}$ siècle, plus largement autoritaire ensuite) ${ }^{6}$. Dans le cas particulier de la France, il faudrait ajouter la division de la paysannerie sur les questions culturelles, religieuses et économiques, qui rend l'électorat rural difficile à capter, y compris pour les partis conservateurs ${ }^{7}$. L'hypothèse peut être affinée par un troisième facteur, évoqué par Daniel-Louis Seiler et Jean-Michel De Waele pour expliquer la montée en puissance des partis agraires dans certains pays d'Europe : l'absence de passé féodal ${ }^{8}$. L'expérience féodale aurait en effet conduit à opposer dès le XVII siècle la noblesse foncière à la bourgeoisie urbaine, qui s'affirme d'abord comme un soutien de l'État absolutiste avant de se rallier, au XIX siècle, aux thèses libérales. Elle contribue ainsi à la bipolarisation de l'échiquier politique en favorisant l'intégration des intérêts agricoles au pôle conservateur, d'abord animé par les anciens notables. En l'absence de passé féodal - par exemple dans les pays scandinaves étudiés par Daniel-Louis Seiler, Danemark exclu -, des partis agrariens peuvent au contraire émerger à la faveur de « l'entrecroisement du clivage "possédants/travailleurs" avec celui opposant monde urbain et monde $\operatorname{rural}^{9}$ ", a fortiori si, comme en Scandinavie, le conservatisme «demeur[e] une force strictement urbaine vouée à la défense de la noblesse d'État dans un premier temps, du monde de l'industrie, de la finance et des affaires ${ }^{10} »$. Dans un pays historiquement féodal et catholique comme la France, la défense des ruraux n'avait que peu de chance d'accéder à l'autonomie politique : elle aurait donc été prise en charge par les autres partis, notamment les partis conservateurs.

Le présent article vise à mettre ces hypothèses à l'épreuve d'un cas particulier, celui de l'échec du parti de l'agriculture lancé par Louis Hervé en septembre 1868, en vue des élections législatives de mai 1869. L'initiative est méconnue. Tentative avortée, le parti de l'agriculture a laissé peu de traces et il avait de toute façon peu d'atouts pour accéder au statut d'objet d'étude: fruit d'une époque généralement considérée comme celle des "proto-partis", il avait été conçu par des hommes de droite réputés réfractaires à l'organisation partisane. L'état des recherches sur le phénomène partisan n'invitait-il pas d'ailleurs à

6. Lipset (S. M.), Rokkan (S.), Structures de clivages, systèmes de partis et alignement des électeurs : une introduction, Bruxelles, Éditions de l'Université de Bruxelles, 2008, p. 88-89 [éd. originale, 1967].

7. Les élections de 1848-1849 en témoignent : les succès conservateurs des élections législatives d'avril 1848 laissent bientôt place à la victoire bonapartiste à l'élection présidentielle de décembre 1848 (qui apparaît dans de nombreuses régions rurales comme un vote d'émancipation à l'égard des anciens notables) puis à la révélation d'une paysannerie rouge dans le Centre notamment mais pas seulement. Cf. Agulhon (M.), La République au village: les populations du Var, de la Révolution à la Seconde République, Paris, Seuil, 1979; Corbin (A.), Archaïsme et modernité en Limousin au XIXe siècle : 1845-1880, Paris, Rivière, 1975, 2 vol. ; Vigier (P.), La Seconde République dans la région alpine : étude politique et sociale, Paris, Presses universitaires de France, 1963, 2 vol. La bibliographie relative à la politisation des paysans au XIX siècle est immense : on ne la sollicitera que ponctuellement, dans la mesure où notre analyse porte essentiellement sur les élites rurales. 8. De Waele (J.-M.), Seiler (D.-L.), "La défense du monde rural : les partis agrariens et paysans », op. cit.

9. Seiler (D.-L.), « Les partis agrariens en Scandinavie », in De Waele (J.-M.), Seiler (D.-L.), dir., Les partis agrariens et paysans en Europe, op. cit., p. 55.

10. Ibid., p. 67. 
classer le dossier sans plus d'analyse comme un exemple parmi tant d'autres de la faiblesse des partis politiques français ? Les déboires du parti de l'agriculture nous semblent au contraire riches d'enseignement. Le contexte était en effet favorable, marqué par la libéralisation relative du régime de Napoléon III, qui encourage la plupart des forces politiques et sociales à approfondir leurs efforts de structuration ${ }^{11}$, et par la montée en puissance, dans le débat public, de l'idée selon laquelle l'avènement du suffrage universel (masculin) annonce la primauté des intérêts matériels sur les questions idéologiques dans la sphère politique $^{12}$. La création en 1867 de la Société des agriculteurs de France, promise à un bel avenir, en témoigne ${ }^{13}$. Son succès contraste avec l'échec du parti de l'agriculture et nous incite à envisager un nouvel élément d'explication qui permet d'articuler le niveau macro-structurel du modèle des clivages à l'échelle plus resserrée de la configuration politique spécifique que connaît la France à l'époque : la concurrence entre deux types d'organisation dans la défense des intérêts ruraux, le premier - le groupe de pression - étant en France, dès l'origine, privilégié sur le second - le parti politique.

C'est dans cette perspective qu'on a analysé les divers documents qui témoignent de la courte vie du parti agricole. La rareté des sources, peu surprenante lorsqu'il s'agit de faire l'histoire d'un échec, et encore moins lorsque ce dernier intervient au stade du projet ${ }^{14}$, a conduit à centrer l'enquête sur les débats suscités par la constitution du parti de l'agriculture dans la presse agricole - La Gazette des campagnes, bien sûr, qui devient pendant quelques mois la tribune du nouveau parti, et les deux revues principales du mouvement rural, le Journal d'agriculture pratique dirigé par Édouard Lecouteux, et le Journal de l'agriculture tenu par Jean-Augustin Barral après son départ du Journal d'agriculture pratique. Mais parce que les discours ne prennent sens que replacés dans le contexte de leur production, on les a rapprochés des rapports de force qui caractérisaient alors le monde rural et l'arène politique où Louis Hervé prétendait faire entrer l'agriculture. Il a ainsi été possible de mieux comprendre l'entreprise de Louis Hervé comme les critiques que lui ont adressées

11. Cf. notamment Nord (P.), The Republican Moment: Struggles for Democracy in Nineteenth-Century France, Cambridge, Harvard University Press, 1995.

12. Sur cette configuration propre au débat public depuis les années 1840, cf. Gaboriaux (C.), La République en quête de citoyens: les républicains français face au bonapartisme rural (1848-1880), Paris, Presses de Sciences Po, 2010. La conviction repose sur l'observation du suffrage censitaire, qui avait mis au centre des débats les enjeux formulés par les notables. On en déduisait logiquement que l'élargissement du suffrage conduirait à l'émergence politique des préoccupations du plus grand nombre. Il en fut en réalité tout autrement : la nécessité de convaincre des électeurs beaucoup plus nombreux a au contraire débouché sur la professionnalisation du métier politique et l'idéologisation des programmes : cf. notamment Garrigou (A.), Le vote et la vertu : comment les Français sont devenus électeurs, Paris, Presses de la FNSP, 1992.

13. Barral (P.), Les agrariens français de Méline à Pisani, Paris, Armand Colin, 1968.

14. Cf. notamment Collovald (A.), «Les poujadistes, ou l'échec en politique », Revue d'histoire moderne et contemporaine, 36 (1), 1989 ; Abélès (M.), L'échec en politique, Belval, Circé, 2005 ; Bock (F.), BührerThierry (G.), Alexandre (S.), dir., L'échec en politique, objet d'histoire, Paris, L'Harmattan, 2008. 
ses contemporains. Si l'analyse des représentations semble confirmer le poids d'une culture politique française globalement hostile aux partis, l'examen du positionnement des différents acteurs tend à montrer que leur réticence à l'égard des partis informe des choix d'ordre stratégique : qu'il s'agisse des élites agricoles, de la paysannerie ou du gouvernement, tous avaient intérêt à favoriser l'organisation d'un groupe d'intérêts au détriment d'un parti.

\section{Un « parti " agrarien}

\section{L'initiative d'un "écrivain agricole "}

Quand Louis Hervé lance l'idée d'un parti de l'agriculture, il est avant tout connu comme l'un des animateurs du mouvement rural. Ce dernier constitue alors un réseau de sociabilité relativement dense, soutenu par des journaux et des revues spécialisées dans les questions agricoles, encadré par des sociétés savantes, des comices et des établissements d'enseignement agricole, et ponctué par un certain nombre d'événements tels les concours agricoles ou les expositions nationales ou universelles. Il a progressivement pris de l'ampleur à partir des années 1840, rejoint par les élites déçues par les changements de régimes successifs. Grands propriétaires terriens - souvent proches du légitimisme mais pas seulement - ou gros fermiers férus d'agronomie issus des «nouvelles couches » rurales et/ou scientifiques, ils ont pour la plupart renoncé à leurs ambitions politiques pour s'investir dans l'exploitation de leurs domaines et plus largement dans le "progrès de l'agriculture »-c'est là le leitmotiv des « écrivains agricoles».

Louis Hervé en fait partie. Né le 13 juin 1814 à Pruillé-l’Éguillé (Sarthe), il grandit dans une famille de cultivateurs sinon fortunée du moins suffisamment aisée pour disposer de deux ou trois domestiques ${ }^{15}$. On le retrouve en septembre 1847 « libraire » (c'est-à-dire aussi et surtout éditeur) à Paris ${ }^{16}$, publiant des auteurs catholiques et monarchistes, souvent légitimistes ${ }^{17}$. Mais $c^{\prime}$ est surtout la vulgarisation des méthodes agronomiques qui l'occupe à partir de la fin des années 1850, avec quelques études et traités ${ }^{18}$ et à La Gazette des campagnes,

15. Registre d'état civil de Pruillé-l’Éguillé, 1814, n² 24, Archives départementales de la Sarthe, 5Mi 266_9. Le recensement de 1836 mentionne les foyers de son grand-père ( « veuf », " propriétaire ») vivant avec une domestique, et de ses parents ( "cultivateurs »), vivant avec son frère de 18 ans et deux jeunes domestiques. Liste nominative de recensement de population de Pruillé-l’Éguillé, 1836, Archives départementales de la Sarthe, 2 Mi 289_130.

16. Bibliographie de la France ou Journal général de l’imprimerie et de la librairie, Paris, Pillet, 1847, p. 544.

17. Entre 1851 et 1867, il publie ainsi les essais de l'abbé Chabau, Antoine Blanc de Saint-Bonnet, Jules Barbey d'Aurevilly, Eugène Loudun, Jean Lander (pseudonyme de Zoé Berthier), Victor Dumax, Henri Lasserre, Eugène Veuillot, Blaise Gisbert, Julie Gouraud, Léon Gautier, Alfred de Perrois, l'abbé Ludovic Chaillot, le comte de Guitaut, Charles-Benjamin Poisson, l'abbé Thorey.

18. Hervé (L.), Mullois (I.), Le livre des habitants des campagnes, Paris, C. Dillet, 1858 (nombreuses rééditions); Hervé (L.), Le sorgho sucré, sa culture comme plante fourragère et comme plante alcoolisable et saccharine. Résumé de tous les essais de culture et résultats industriels obtenus depuis l’introduction de cette plante 
dont il est l'un des rédacteurs dès sa création et qu'il refonde en 1864 après avoir été inquiété pour l'un de ses articles sur les élections de 1863, paru dans Le Journal des villes et des campagnes et sanctionné d'un avertissement du gouvernement. Le caractère exclusivement agricole de l'hebdomadaire parait le prémunir contre les chausse-trapes de la politique : «Tout pour les campagnes et par les campagnes ", indique sa devise. Il lui permet également d'échapper à la demande d'autorisation préalable, au cautionnement et au droit de timbre, par lesquels le gouvernement contrôle la presse politique. On y trouve des informations sur les dernières avancées agronomiques, la situation économique des campagnes, les événements que sont les concours agricoles, les foires ou les dernières publications en la matière. En 1860, La Gazette des campagnes compte 12000 abonnés ${ }^{19}$, ce qui est tout à fait honorable (à titre de comparaison, Le Figaro tourne alors autour de 15000 abonnés) et même un peu mieux que son principal concurrent, Le Journal d'agriculture pratique (environ 10000 abonnés), dont elle se distingue par sa tonalité réactionnaire. Le très satirique Jean-François Vaudin la décrit ainsi comme "prêch[ant], avec des pudeurs de feuille cléricale et en termes choisis, en dehors de son idée industrielle, contre la littérature des chiffons, les falbalas, les fanfreluches, les épluchures de musique, les poupées, les polkas, qui font naître la concupiscence, contre les cabinets de lecture, ces déversoirs de turpitudes et d'imbécillités, les romans de Mme Sand, les femmes émancipées ${ }^{20}{ }$.

Louis Hervé y exprime en réalité son intérêt pour la politique, en des termes qui ne disent rien de ses préférences politiques, même si les rubriques consacrées à l'éloge de la civilisation rurale et chrétienne permettent de les deviner aisément. "L'éducation rurale touche à l'homme public et privé dans toutes les sphères de l'activité ", affirme-t-il dès novembre $1864^{21}$. "La vie rurale est de plus en plus mêlée directement aux grandes affaires du pays ", redit-il en décembre de la même année ${ }^{22}$. En 1865, il ose même - prudemment - évoquer les élections municipales : "Sans nous immiscer dans les questions qui sont du ressort des journaux politiques, nous restons pleinement dans notre programme en invitant de toutes nos forces nos lecteurs à montrer, dans cette occasion, une parfaite intelligence de leurs droits et de leur tâche d'électeurs, en n'envoyant aux conseils des communes rurales que des hommes d'un esprit éclairé, d'un caractère ferme et conciliant, et surtout des hommes capables, par leur savoir agronomique, de donner une heureuse impulsion à l'administration, et de tirer parti des ressources que possède chaque commune, pour les

\footnotetext{
en France, Paris, A. Goin, 1858 ; Hervé (L.), dir., L’almanach des campagnes, Paris, s.n., 1860 ; Hervé (L.), Les orphelinats agricoles en France, Paris, bureaux du Contemporain, 1868.

19. L'abonnement est alors le seul moyen de diffusion autorisé hors de la ville d'édition.

20. Vaudin (J.-F.), Gazetiers et Gazettes : histoire critique et anecdotique de la presse parisienne, Paris, chez tous les libraires, 1860, p. 104-105.

21. Hervé (L.), "Chronique générale », La Gazette des campagnes, 26 novembre 1864, p. 377.

22. Hervé (L.), «Chronique générale », La Gazette des campagnes, 10 décembre 1864, p. 393.
} 
appliquer aux progrès de l'instruction et de la prospérité générale ${ }^{23}$. Il récidive en août $1867^{24}$, à l'occasion du renouvellement des conseils généraux, déplorant parmi les élus le peu d' "agriculteurs" (à l'époque le terme désigne un membre de l'élite rurale, « celui qui fait valoir par lui-même et en grand » selon le Larousse ${ }^{25}$ ). Ses lecteurs n'ont donc sans doute pas été surpris d'apprendre dans le numéro du 5 septembre 1868 la mue de son journal agricole en journal politique (ce qui implique alors déclaration au ministère de l'Intérieur, cautionnement au Trésor, droit de timbre - formalités dont les journaux non politiques sont exemptés) et la formation d'un " parti de l'agriculture ${ }^{26}$.

Le raisonnement est simple : défendre l'agriculture, c'est parler au nom de l'électorat paysan, alors ultra-majoritaire ${ }^{27}$ et $s^{\prime}$ assurer par conséquent de faire élire ses candidats. On reconnaît là les fondamentaux du discours agrarien. La diversité et les divisions du monde rural sont occultées, et les intérêts des populations qui résident dans les campagnes identifiés à ceux de "l'Agriculture ». Cette dernière est en effet érigée au rang de science pratique unifiée, que seuls les gros exploitants et les agronomes sont susceptibles de comprendre et de défendre ${ }^{28}$. Émancipés des féodalités anciennes, les habitants des campagnes retrouvent ainsi leurs "guides naturels ", désignés non plus par la naissance mais par la compétence ${ }^{29}$.

\section{Un contexte apparemment favorable}

La libéralisation de l'Empire donne au projet de Louis Hervé un certain nombre d'atouts. Progressivement mise en place dans les années 1860, elle est particulièrement perceptible à la fin de la décennie. Assouplissement du régime de la presse (loi du 11 mai 1868), tolérance à l'égard des réunions électorales

23. Hervé (L.), « Chronique générale », La Gazette des campagnes, 15 juillet 1865, p. 218.

24. Hervé (L.), «Chronique générale », La Gazette des campagnes, 17 août 1867, p. 1089.

25. "Agriculteur », in Larousse (P.), Grand dictionnaire universel du XIX siècle, Paris, Administration du grand dictionnaire universel, 1866-1877, tome 1, p. 142.

26. Hervé (L.), « La gazette des campagnes politiques », La Gazette des campagnes, 5 septembre 1868, p. 281.

27. En 1856, la population vivant de l'agriculture est estimée à 19140000 personnes, soit 53,1\% de la population totale, et $70 \%$ des Français vivent à la campagne. Plessis (A.), De la fête impériale au mur des fédérés : 1852-1871, Paris, Seuil, 1979, p. 140.

28. À cet égard, l'entreprise ne saurait être considérée comme le pendant agricole des candidatures ouvrières des années 1860 : alors que des ouvriers affirment à travers le Manifeste des soixante (1864) leur droit et intérêt à être représentés par des ouvriers, les élites agricoles que nous étudions ici mettent au contraire en avant leur supériorité sociale, intellectuelle et morale à l'égard du peuple des campagnes pour s'en autoproclamer unilatéralement les porte-parole.

29. Sur ces questions, cf. Barral (P.), Les agrariens français de Méline à Pisani, Paris, Armand Colin, 1968 ; et, dans une perspective marxiste très critique à l'égard du précédent, à qui il reproche d'avoir donné, à travers le concept d'agrarisme, une caution scientifique au mythe bourgeois de l'unité du monde rural: Gratton (P.), Les luttes de classes dans les campagnes, Paris, Anthropos, 1971 et Les paysans français contre l'agrarisme, Paris, Maspero, 1972. Les travaux plus récents tendent à dépasser l'opposition : Cornu (P.), Mayaud (J.-L.), dir., Au nom de la terre : agrarisme et agrariens en France et en Europe du XIX siècle à nos jours, Paris, La Boutique de l'histoire, 2007. 
(6 juin 1868), relâchement des pressions administratives ${ }^{30}$ : autant d'éléments qui conduisent les membres de l'élite rurale à renouer avec les ambitions politiques qui avaient pu animer certains d'entre eux dans les années 1840, du moins à tenter de regagner un rôle politique à l'échelon local voire national ${ }^{31}$. En 1862, La Gazette des campagnes, alors dirigée par l'abbé Mullois, renonce ainsi à l'exemption du timbre et du cautionnement dont bénéficient à l'époque les publications agricoles qui s'engagent à ne pas traiter des questions politiques : rebaptisée Courrier universel, la revue est autorisée au mois de mai à " parler des choses de la politique et de l'économie sociale ${ }^{32} »$. En février 1867, le Journal d'agriculture pratique décide à son tour de subir le timbre et le cautionnement. Avec la loi du 11 mai 1868, qui supprime l'autorisation préalable en matière de presse au profit de la simple déclaration préalable, d'autres suivent, et en particulier La Gazette des campagnes, que Louis Hervé a refondée en 1864.

L'enquête agricole lancée en 1866, alors même que l'agriculture connaît des difficultés économiques, donne en outre aux élites rurales l'occasion de se faire entendre au-delà des sociétés savantes et des conseils municipaux ou généraux, jusque dans la presse parisienne et l'enceinte parlementaire. Elle suscite la rédaction d'un " programme » agricole, porté par le mouvement rural et relayé dans les journaux parisiens, qui réclame l'allègement des charges de toute nature pesant sur l'agriculture - impôts, taxes et autres droits de circulation, recrutement militaire, tarifs de procédure civile - et que Louis Hervé reprend à son compte au début de l'année 1869. L'enquête agricole semble même avoir permis de faire émerger une personnalité qui soit capable de prendre la tête du parti agricole - c'est en tout cas ce qu'imagine Louis Hervé qui, quelques semaines avant les élections, en appelle à Léonce de Lavergne. Passé par le Conseil d'État et député sous la Monarchie de Juillet, ce dernier a renoncé - provisoirement - à la politique sous la République et le Second Empire pour se spécialiser dans l'économie rurale, ce qui lui a valu de devenir membre de l'Académie des sciences morales et politiques en 1855 . Après un bref retour en politique - le temps d'échouer aux élections législatives de 1863 contre le candidat officiel -,

30. Alain Plessis note ainsi que, dès 1863, «les préfets, et plus encore les maires, les fonctionnaires et les curés témoignent de moins de zèle que précédemment pour les candidats du gouvernement, et ces derniers tiennent à affirmer leur indépendance ». Plessis (A.), De la fête impériale..., op. cit., p. 209. Les contrôles demeurent: Faure (A.), "Origine et usage d'une liberté très surveillée », in Dalotel (A.), Faure (A.), Freiermuth (J.-C.), dir., Aux origines de la Commune: le mouvement des réunions publiques à Paris 18681870, Paris, Maspero, 1980, p. 21. Mais l'effervescence politique des élections de 1869 apparait néanmoins comme une rupture, qui débouche d'ailleurs sur la montée en puissance de l'opposition au Corps législatif : Cossart (P.), Le meeting politique. De la délibération à la manifestation (1868-1939), Rennes, Presses universitaires de Rennes, 2010.

31. J'ai étudié les aléas de leurs stratégies politiques dans «Entre les innovations agronomiques et les pratiques paysannes, la figure de l'agriculteur pratique au XIX e siècle », in Bonneuil (C.), Denis (G.), Mayaud (J.-L.), dir., Sciences, chercheurs et agriculture : pour une histoire de la recherche agronomique, Versailles-Paris, Quae-L'Harmattan, 2008.

32. Guérin (M.), « Le courrier universel », La Gazette des campagnes, 19 avril 1862, n51, p. 613. 
il s'est beaucoup exprimé sur l'enquête agricole et incarne ainsi, aux yeux de Louis Hervé, « le candidat providentiel du grand parti agricole ${ }^{33}$ ».

Le projet de Louis Hervé differre ainsi peu des « partis » tels qu'on les connaît à l'époque, sous un régime globalement hostile aux associations ou réunions politiques. Il s'agissait pour lui de fédérer un réseau plus ou moins informel autour de son journal, La Gazette des campagnes, destiné à en diffuser le programme et les mots d'ordre, et de transformer le mouvement rural, qui avait jusqu'ici limité son champ d'action aux questions économiques, en force politique capable de faire élire ses députés au Corps législatif, à l'aide de comités électoraux et sous la direction d'un chef charismatique. L'initiative de Louis Hervé est en effet révélatrice du fonctionnement de ces groupes informels, déjà conçus comme des entreprises de conquête des suffrages mais pas encore dotés de ces structures organisationnelles pérennes et complètes qui caractérisent aux yeux de Joseph La Palombara et Myron Weiner les partis au sens moderne du terme ${ }^{34}$. Ce que souhaite en effet Louis Hervé, c'est fonder un parti susceptible de rivaliser avec le parti libéral et le parti républicain. Or ces partis, remarque$\mathrm{t}$-il, se servent des candidatures multiples pour imposer des personnalités qui leur permettent à la fois d'exister sur la scène politique et de discipliner leurs troupes, palliant ainsi les risques de dispersion inhérents à leur caractère informel :

«La logique et le bon sens politique dictent cette tactique aux divers partis. Ces élections multiples consacrent l'influence des candidats comme chefs de leur école; et chaque école profite de ce moyen pour s'organiser, se discipliner et donner la mesure totale de sa puissance. »

Il faut donc selon lui que le parti agricole fasse de même, "pour affirmer ses doctrines et ses prétentions, et pour acquérir la force de cohésion si nécessaire aux intérêts qu'il représente». Et si Léonce de Lavergne lui semble tout indiqué pour devenir le chef du parti agricole, c'est que ses compétences scientifiques et ses talents d'orateur en font l'égal d'Adolphe Thiers, et même «le Thiers de l'Avenir », un Adolphe Thiers qui ne se contenterait pas de liquider le gouvernement personnel mais poserait les principes politiques de la société à venir ${ }^{35}$. Immense prétention et/ou naïveté extrême ? Depuis qu'il a lancé l'idée d'un grand parti de l'agriculture en vue des élections de 1869, Louis Hervé a reçu de nombreux soutiens, ce qui explique en partie son enthousiasme. Dès le 26 septembre 1868, soit trois semaines après le cri de ralliement paru dans La Gazette des campagnes, il peut ainsi se féliciter d'avoir l'appui d'une large partie du mouvement rural: «Des hommes politiques éminents, des députés,

33. Hervé (L.), «Les candidats généraux et locaux », La Gazette des campagnes, 8 mai 1869, n 33, p. 139.

34. LaPalombara (J.), Weiner (M.), eds, Political Parties and Political Development, Princeton, N.J., Princeton University Press, 1966.

35. Hervé (L.), « Les candidats généraux et locaux », op. cit. 
des sociétés agricoles, des propriétaires, des fermiers; en un mot l'agriculture militante, l'esprit rural, à tous les degrés, nous promettent un appui, un concours énergique ${ }^{36}$. » La liste des parrains de l'entreprise, que l'éditeur de La Gazette des campagnes, Charles Blériot, fait paraître début décembre dans l'une de ses publications périodiques, L'Ouvrier, compte en effet des personnalités prestigieuses : Léonce de Lavergne, le marquis d'Andelarre, Edmond de Tillancourt, le vicomte de Tocqueville (frère d'Alexis), le marquis de Vogué, Paul de Gasparin, Paul Bethmont, Louis Buffet ou encore Claude-Marie Raudot ${ }^{37}$. Dès le mois d'octobre, les deux principales revues agricoles du moment avaient d'ailleurs salué la naissance du parti agricole. « Nous souhaitons de tout cour le plus grand succès à M. Hervé ", note ainsi Jean-Augustin Barral, avant d'ajouter : «Les habitants des campagnes commencent à comprendre qu'ils doivent intervenir davantage dans les affaires de l'État, parce que les affaires de l'agriculture en iront beaucoup mieux ${ }^{38}$. » Édouard Lecouteux l'avait précédé, mais dans une formule où transparaissait l'agacement du concurrent doublé : « La Gazette des campagnes peut croire qu'elle ne sera pas seule à tâcher que la Société des agriculteurs de France, créée par l'initiative du Journal d'agriculture pratique, réalise les espérances qui ont accompagné et favorisé ses heureux débuts ${ }^{39}$. » Et en avril 1869, Louis Hervé se réjouit de voir des comités électoraux se former selon ses vœux, comme à Quimper par exemple ${ }^{40}$.

L'initiative de Louis Hervé apparaît ainsi à ce stade largement influencée par le contexte politique. Alors que la répression avait jusqu'ici favorisé l'investissement des anciennes et nouvelles élites agricoles dans des activités présentées comme apolitiques, son relâchement à la fin des années 1860 suscite chez certaines d'entre elles l'espoir d'un retour sur la scène politique, selon des moyens analogues à ceux des autres forces en présence (journaux, réseaux de sociabilité, candidatures multiples, etc.).

\section{Les réticences des élites agricoles : la hantise des partis ?}

\section{Parti ou groupe de pression : un malentendu?}

Peu à peu pourtant, et dès l'automne 1868, Louis Hervé perd ses soutiens les plus en vue. À la fin du mois de novembre, Barral fait ainsi machine arrière :

«Tout récemment, écrit-il, un de nos confrères a pensé qu'il fallait créer un journal agricole absolument politique. Nous avons souhaité bonne chance à

\footnotetext{
36. Hervé (L.), « La gazette des campagnes politiques », op. cit.

37. L'Ouvrier, 5 décembre 1868 , p. 256.

38. Barral (J.-A.), «Chronique agricole (première quinzaine d'octobre 1868) », Journal de l'agriculture, 1868 , t. 4 , p. $158-159$.

39. Lecouteux (E.), "L'agriculture et les prochaines élections générales », Journal de l'agriculture pratique, 1868 , t. 2, p. 483.

40. Hervé (L.), « Élections », La Gazette des campagnes, 3 avril 1869, p. 106.
} 
son entreprise et salué sa bienvenue. Cependant, il ne nous paraît pas exact de dire qu'il y a un parti agricole ${ }^{41}$.»

Et de préciser les points d'achoppement. Pour lui, les intérêts agricoles doivent certes être défendus sur le terrain politique mais ils n'ont pas pour autant vocation à susciter la création d'un parti. Au contraire, l'objectif est d'encourager les mesures favorables à l'agriculture quel que soit le parti qui les mette en avant. Si le Journal de l'agriculture prend régulièrement position à l'occasion des élections, ce n'est donc pas pour soutenir tel ou tel parti, fût-il agricole, mais pour contraindre les candidats de tous les partis à s'engager en faveur de l'agriculture :

«Lorsque nous avons demandé qu'il y eût dans les assemblées délibérantes et législatives plus d'hommes voués à l'agriculture, nous avons entendu émettre le vœu que les électeurs tinssent davantage compte des aptitudes agricoles des candidats, et nous croyons que les affaires du pays seraient mieux dirigées, si les hommes qui sont au pouvoir ou dans l'opposition étaient davantage agriculteurs, mais il y a loin de là à vouloir exclure les représentants des autres grands intérêts du pays. »

De même, Barral tient à s'expliquer sur l'éloge des candidats indépendants qu’on lit fréquemment sous sa plume :

«Nous n'apercevons pas le moins du monde pour quelle raison le gouvernement impérial ne pourrait pas, sur tous les points, donner satisfaction aux besoins de l'agriculture nationale. Il a déjà commencé par la nouvelle loi sur les chemins vicinaux et par une impulsion de quelque valeur donnée à l'instruction agricole. Mais il lui reste énormément à faire. S'il agit, il méritera d'avoir le concours des hommes indépendants. S'il prend des mesures contraires à des intérêts si graves, il encourra justement leur opposition. Voilà comment il faut selon nous entendre la pratique de la vie rurale dans ses rapports avec la politique $^{42}$.»

Défense d'intérêts sectoriels sans ambition gouvernementale : Barral n'emploie pas le mot, mais ce qu'il décrit a tout de ce qu'on appellerait aujourd'hui un groupe de pression. Cette définition des rapports de l'agriculture à la politique n'est pas nouvelle chez lui qui, dans les colonnes du Journal d'agriculture pratique puis à la tête du Journal de l'agriculture, s'est toujours montré soucieux de présenter l'agriculture comme un «terrain neutre» susceptible de rallier les bonnes volontés d'où qu'elles viennent. On peut en revanche s'étonner de son enthousiasme initial pour le parti agricole, qui supposait au contraire de rompre avec la ligne de conduite à laquelle il s'était jusqu'ici tenu.

41. Barral (J.-A.), «Chronique agricole (première quinzaine de novembre 1868) », Journal de l'agriculture, année 1868, t. 4, p. 434.

42. Ibid., p. 435. 
S'agirait-il d'un malentendu, formé à partir de l'équivocité du mot " parti » ? Ce dernier, nous dit Pierre Larousse, signifie alors «l'union de plusieurs personnes contre d'autres qui ont un intérêt, une opinion contraire ${ }^{43} »$. Il peut donc recouvrir la double acception de "force politique " et de "groupe de pression ». Aux côtés de "parti libéral » ou "parti républicain", on trouve ainsi encore fréquemment "parti des libre-échangistes ", "parti des protectionnistes ", et, péjorativement, "parti boursier». Or "parti agricole», sans être très fréquent, avait surtout été employé jusqu'ici au sens de "groupe de pression ». C'était sous cette dernière acception que l'entendaient en 1846 un certain nombre d' " agriculteurs », qui, autour d'Alix Sauzeau, avaient tenté de " fonder le parti agricole », conçu comme le « défenseur officieux » de l'agriculture et chargé « de recueillir toutes les réclamations, toutes les observations qui afflueraient de toutes parts, de suivre tous les travaux des pouvoirs législatifs et de signaler les erreurs dont ils fourmillent ${ }^{44}{ } »$.

Dans les années 1860, les animateurs du mouvement rural étaient d'ailleurs tout disposés à ne voir dans le parti agricole que le prolongement du combat qui avait été le leur durant tout le Second Empire : rallier les hommes de tous horizons à la cause de l'agriculture pour la voir mieux traitée dans les assemblées et au gouvernement. C'était le mot d'ordre de la plupart des revues agricoles, c'était l'objectif que venait de se fixer la Société des agriculteurs de France, que Lecouteux avait imaginée tout à la fois groupe de pression et organisation professionnelle. Elle devait selon lui :

« D’une part, reprendre l'œuvre interrompue du congrès central de Paris, où, chaque année, les délégués des sociétés et comices agricoles venaient exprimer les vues et les besoins de leurs mandataires et se livrer à des discussions qui, pour la plupart, ont servi de base aux meilleures mesures administratives - et, d'autre part, fonctionner à l'instar des grandes sociétés d'Angleterre et d'Allemagne, qui joignent l'action à la parole, fondent des prix, instituent des enquêtes et des concours, cherchent enfin par tous les moyens, notamment par leurs concours tenus à tour de rôle dans les diverses régions du pays, à développer partout l'esprit d'initiative locale et à centraliser les forces agricoles ${ }^{45}$. »

Le passé de Louis Hervé militait d'ailleurs en faveur de cette interprétation - erronée - du parti agricole qu'il souhaitait constituer. N'avait-il pas refondé La Gazette des campagnes pour la réinscrire dans la presse agricole, quand son premier associé, l'abbé Mullois, avait au contraire décidé de la transformer, sous un autre nom, en journal politique ? Surtout, il s'était fait connaître par ses engagements protectionnistes, dans une discussion où l'expression

\footnotetext{
43. Larousse (P.), « Parti », op. cit., t. 12, p. 332.

44. Sauzeau (A.), Les paysans ou la politique et l'agriculture, Paris, Vve Bouchard-Huzard, 1849, p. 263 et 266.

45. Lecouteux (E.), «La société des agriculteurs de France », Journal d'agriculture pratique, année 1868, t. 1, p. 354 .
} 
«parti agricole » signifiait « groupe de pression » : elle désignait alors les défenseurs des intérêts agricoles que les partisans du libre-échange s'efforçaient de détacher du protectionnisme ${ }^{46}$.

Léonce de Lavergne semble s'y être lui-même trompé. Inspirateur du projet de Louis Hervé, qui s’appuie sur ses analyses des résultats de l'enquête agricole pour constituer son programme ${ }^{47}$, il en est aussi l'un des premiers soutiens. Mais quand Louis Hervé l'appelle à prendre la tête du parti agricole, au même titre que Thiers pour le parti libéral, il se dérobe : «Mes idées personnelles suivent un tout autre cours », lui écrit-il, invoquant les «divisions» du parti libéral, l'« état de sa santé » et l'« habitude d'une retraite studieuse qui [lui] suffit parfaitement ». Et la fin de sa lettre souligne la différence d'appréciation qui le sépare de Louis Hervé quand il s'agit du " parti agricole » : «On peut, Dieu merci, servir notre cause commune sans être député ${ }^{8}$ ", conclut-il. Quand Louis Hervé avait fondé le parti agricole sur la conviction que le mouvement rural devait désormais s'affirmer comme une force politique à part entière s'il voulait voir ses revendications satisfaites, Lavergne laisse au contraire entendre que la cause de l'agriculture peut être défendue en dehors de la compétition électorale.

Avant même la tenue des élections, Louis Hervé doit ainsi reconnaître son échec. "Cette fois encore, note-t-il à la veille du scrutin, l'élément rural gravitera, dans ses votes, dans l'orbite de plusieurs partis, qui se serviront plus de lui qu'il ne se servira d'eux ${ }^{49}$. » De fait, les candidats qui se réclament exclusivement du parti agricole sont rares et plus rarement encore élus : la plupart des quatre-vingts « députés agricoles » mentionnés par Lecouteux au lendemain du scrutin n'ont fait que reprendre le programme du parti de l'agriculture sans renoncer pour autant à défendre les couleurs du gouvernement ou de l'Union libérale, plus rarement du légitimisme ou de la République ${ }^{50}$. Louis Hervé tire bientôt les leçons de ce revers :

«Pour nous, qui sommes un parti encore dans la période d'incubation, nous ne pouvons compter comme une épreuve sérieuse les timides essais de nos amis les plus hardis. Notre heure n'est pas venue. Il nous faut quelques années d'efforts et de travaux pour nous constituer avec un programme parfaitement défini et en lui-même et dans ses rapports avec les idées sur lesquelles les partis actuels basent leurs espérances ${ }^{51}$.»

\footnotetext{
46. Cf. par exemple le compte rendu des réunions de la Société d'économie politique consacrées à la question de l'échelle mobile des droits sur les blés, Journal des économistes, avril-juin 1859, t. 22, p. 147-154.

47. Cf. par exemple Lavergne (L. de), «L'enquête agricole », Revue des deux mondes, 15 novembre 1868, t. 78 , p. $400-427$.

48. Lettre publiée sous le titre "Une lacune dans les candidatures», Gazette des campagnes, 15 mai 1869, p. 154.

49. Hervé (L.), « Le scrutin du 23 mai », La Gazette des campagnes, 22 mai 1869, p. 161.

50. Lecouteux (E.), «Chronique agricole (10 juin 1869) », Journal d'agriculture pratique, année 1869, t. 1, p. 827-828.

51. Hervé (L.), « Le parti agricole aux élections », La Gazette des campagnes, 5 juin 1869, p. 177.
} 
Là semble en effet résider le malentendu : doté d'un programme exclusivement agricole, le parti de l'agriculture a été perçu par la plupart de ses soutiens politiques comme un groupe de pression qu'il était important de ne pas s'aliéner à l'heure des élections, et non comme une force politique intégrant la compétition électorale et modifiant à ce titre la configuration de l'échiquier politique.

\section{Le rejet de l'" esprit de parti "}

La désaffection des élites rurales reste néanmoins surprenante: la fondation du parti agricole rompait certes avec la défense des intérêts agricoles telle qu'elles l'avaient conçue jusqu'ici, mais elle leur aurait pourtant offert l'occasion de réaliser les ambitions politiques qu'elles affichaient désormais. Depuis le début des années 1860, Barral et Lecouteux n’avaient pas cessé d'encourager les candidatures exclusivement agricoles, ne répugnant pas d'ailleurs à se porter eux-mêmes candidats à la députation, de sorte qu'ils avaient pu donner justement l'impression de vouloir convertir le mouvement agricole en structure partisane. Pourquoi dès lors prennent-ils leurs distances avec Louis Hervé ? À lire les réactions des uns et des autres, il semble que le mot de "parti », employé par Louis Hervé pour décrire les aspirations politiques du mouvement rural, ait cristallisé leurs réticences. "À vrai dire, note d'abord Lecouteux, ce titre de parti agricole ne nous va pas beaucoup ${ }^{52}$ ", avant d'affirmer deux semaines plus tard que Louis Hervé et ses amis ont « le tort très grave de se constituer à l'état de $\operatorname{parti}^{53} »$.

Leur argumentation témoigne de la hantise des partis propre à la France, mise en avant par G. Lavau. L'inconvénient du parti à leurs yeux, c'est en effet d'abord qu'il se donne une «mission spéciale » qui le détourne de l'intérêt général : faire « œuvre de parti», ce n'est pas faire « œuvre nationale» selon Lecouteux ${ }^{54}$. Sous la plume des défenseurs autoproclamés de l'agriculture, l'objection peut paraître incongrue : n'ont-ils pas eux-mêmes voué leurs efforts à la promotion d'intérêts économiques et sociaux particuliers? N'est-ce pas justement au nom de leur vocation «spéciale » qu'ils ont appelé les leurs à déserter la "politique générale ${ }^{55} »$ ? Lecouteux comme Barral ont anticipé l'objection. Les chroniques qu'ils publient à l'automne 1868 visent à démontrer la vocation générale de la défense des intérêts agricoles. "L'intérêt agricole, rappelle Lecouteux, est, en France, l'intérêt essentiel de la population la plus nombreuse ", il est en outre intimement lié à celui de l'industrie et du commerce: "Sans une certaine réduction du prix de revient des produits du sol, la France ne peut pas espérer

52. Lecouteux (E.), "Chronique agricole (5 novembre 1868) », Journal d'agriculture pratique, 1868, t. 2, p. 602.

53. Lecouteux (E.), «Le parti de l'agriculture », Journal d'agriculture pratique, 1868, t. 2, p. 641.

54. Ibid., p. 641 et 644.

55. Ibid., p. 641. 
de prospérité manufacturière et commerciale. Donc le progrès agricole est la première solution de tous nos problèmes économiques ${ }^{56}$. " Comme l'écrit Barral, qui reprend la formule à Lavergne, l'agriculture par conséquent «ne demande rien qui ne soit conforme à l'intérêt général ${ }^{57}$ ».

Se situer sur le terrain économique et social, y compris lorsqu'ils s'aventurent dans l'arène politique, leur permet en outre et surtout, disent-ils, de dépasser le conflit propre au politique pour placer leur combat sous l'autorité consensuelle de la science et de la raison. La forme partisane évoque immanquablement chez eux «le déchaînement des passions politiques" (Lecouteux) et la « guerre » (Barral) des groupes sociaux et des opinions. Ils refusent ainsi d'être des «hommes de parti » ou de céder à quelque degré que ce soit à l'" esprit de parti », qui, en ce siècle troublé, sont souvent synonymes d'intolérance, de mépris, de haine de l'autre. Le Grand dictionnaire universel du XIXe siècle définit alors en effet l'" homme de parti» comme un «homme qui se montre passionné en tout ce qui concerne son parti, et injuste en tout ce qui concerne le parti opposé », l'« esprit de parti » étant quant à lui décrit comme la "disposition qui porte au mépris ou à la haine des idées qu'on ne partage pas, des personnes qui sont d'un autre parti ${ }^{58} »$. La mission des agriculteurs militants est tout autre selon Barral :

«Lorsqu'une question se présente, cette question fût-elle politique, ils doivent l'apprécier, la juger et la résoudre selon l'influence qu'elle peut exercer sur la prospérité agricole du pays. Ils doivent savoir approuver une bonne mesure et en blâmer une mauvaise, lors même que l'auteur de cette mesure appartienne à leur propre parti politique ».

Pour Barral, les questions agricoles sont en effet soumises au régime de la vérité, qui permet de départager nettement les «bonnes» des «mauvaises » mesures, tandis que les questions politiques, rapportées aux « convictions » des uns et des autres, restent en droit indécidables et ne se règlent en fait qu'en fonction des rapports de force. Au siècle des révolutions, la défense des intérêts agricoles présente au contraire l'immense avantage d'engendrer immanquablement la «conciliation, qui, ajoute Barral, dans la pratique de la vie, fait beaucoup plus de bien, parce qu'elle ne laisse jamais après elle l'amertume de la défaite et les regrets des violences ».

À plus d'un siècle de distance, les élites agricoles semblent ainsi rêver à la « fin des idéologies ", au sens que les débats des années 1980 ont parfois assigné à la formule de Raymond Aron, non sans la détourner de sa signification originelle. Ce que Lecouteux appelle alors «nouvel ordre économique » avant d'adopter,

\footnotetext{
56. Ibid., p. 642. C’est moi qui souligne.

57. Barral (J.-A.), « Chronique agricole (première quinzaine de novembre 1868) », op. cit., p 435.

58. Larousse (P.), « Parti », op. cit.
} 
dans les années 1880, l'expression "politique des intérêts matériels ${ }^{59}$ ", désigne un monde où l'avènement des masses en politique mettrait tout le monde d'accord sur les fins de la société démocratique - le bien-être matériel du plus grand nombre -, imposant dans les débats les questions économiques et dans la compétition pour le pouvoir les plus capables en la matière.

On comprend dès lors leurs réticences à l'égard de l'entreprise de Louis Hervé. En cherchant à fonder un parti de l'agriculture capable de rivaliser avec l'Union libérale ou le parti républicain, le directeur de La Gazette des campagnes acceptait d'entrer dans le conflit politique que les rédacteurs du Journal d'agriculture pratique et du Journal d'agriculture cherchaient justement à dépasser. Le vocabulaire de ses chroniques en témoigne, qui multiplient les appels au « grand combat» en destination des «croisés du grand parti de l'agriculture ${ }^{60}$ ». Les troupes lui ont pourtant fait défaut. Imprégnés de la «culture politique de la généralité » décrite par Pierre Rosanvallon ${ }^{61}$ plus que du souci de l'abstraction dénoncé par Georges Lavau ${ }^{62}$, les animateurs du mouvement rural n’y ont vu que le germe de la division et de la violence.

\section{Les raisons d'un échec}

\section{Le spectre de la réaction}

Sans remettre en cause la réalité de la hantise des partis qui habite une bonne partie des élites politiques de l'époque, il est néanmoins important de s'interroger sur les enjeux de son usage en discours. Comme le rappelle très justement Michel Offerlé, le «label parti» n'a cessé en effet de «faire partie de l'enjeu de la compétition politique et scientifique ${ }^{63} »$. Il se pourrait donc qu'en invoquant contre Louis Hervé l'" esprit de parti ", Barral et Lecouteux n'expriment pas seulement leur conception de la défense de l'agriculture ou de la politique en général, mais qu'ils brandissent un argument efficace dans un conflit qu'il nous faut désormais analyser. À cet égard, il faut reconnaître que l'entreprise de Louis Hervé, qu'elle relève ou non de la forme partisane, avait tout pour les inquiéter. À la tête du Journal d'agriculture pratique depuis 1850, Barral en avait été écarté en 1866 à la mort de son fondateur, Alexandre Bixio, avec qui il avait fréquenté les milieux fouriéristes sous la Seconde République. Remplacé par Lecouteux, que les héritiers de Bixio avaient dû trouver moins subversif, il avait

\footnotetext{
59. Lecouteux (E.), «La politique des intérêts matériels », Journal d'agriculture pratique, année 1881, t. 1, p. 114-116.

60. Hervé (L.), «La gazette des campagnes politiques », 5 et 26 septembre 1868, op. cit.

61. Rosanvallon (P.), Le modèle politique français : la société civile contre le jacobinisme de 1789 à nos jours, Paris, Seuil, 2004, p. 13.

62. Lavau (G.), Partis politiques et réalités sociales..., op. cit., p. 139.

63. Offerlé (M.), « Qu'est-ce qu’un parti politique en France au XIXe siècle ? », in Saint-Gille (A.-M.), dir., Cultures politiques et partis aux XIX ${ }^{e}$ et XX $X^{e}$ siècles : l'exemple allemand, Lyon, Presses universitaires de Lyon, 2005.
} 
fondé le Journal de l'agriculture, dont il voulait faire « une revue plutôt qu'un journal, revue qui seule admet les études longues et approfondies ${ }^{64} »$. Les chroniques des deux revues témoignent des rivalités et divergences qui l'opposent à Lecouteux, même s'il adhère à la Société des agriculteurs de France dès sa création. En fondant un parti agricole, Louis Hervé s'imposait ainsi comme un nouveau concurrent, dont les ambitions hégémoniques ne pouvaient plaire aux deux publicistes. Plus gênant encore aux yeux de Barral et Lecouteux, il cherchait à enrôler le mouvement agricole sous un drapeau politique quaucun des deux écrivains agricoles ne pouvait approuver. Le rejet de l'« esprit de parti » cache mal leur désaccord sur le fond. Quoiqu'unanimes à défendre des candidatures indépendantes et exclusivement agricoles, les trois hommes appartenaient en effet à des familles politiques très différentes. Très marqué à droite, Louis Hervé effraie à la fois Barral et Lecouteux.

Saluant le parti de l'agriculture alors qu'il pensait encore que l'entreprise relevait du groupe de pression, Barral avait déjà émis quelques réserves : « Nous lui reprocherions peut-être de couvrir et de protéger la droite, au détriment du centre et surtout de la gauche, mais à cela près, nous proclamons toute l'utilité de son entreprise ${ }^{65}$.» Une fois le malentendu levé, il n'est plus question de soutenir le parti agricole. La méfiance semble d'ailleurs réciproque. La candidature de Barral en Moselle, comme celle de Pierre Joigneaux en Côte d'Or est soutenue du bout des lèvres par Louis Hervé, qui les trouve décidément trop à gauche : "Malheureusement, il faut le dire, nos deux honorés confrères ont dans le passé des attaches politiques qui leur aliènent de nombreux électeurs ", note-t-il à la veille des élections ${ }^{66}$. La position de Lecouteux est sensiblement différente. Quand Barral refuse de se mêler au jeu des partis, il semble au contraire avoir été séduit par l'idée du parti agricole. Plus proche que Barral des quelques libéraux qui, comme Léonce de Lavergne, ont dans un premier temps soutenu l'entreprise de Louis Hervé, il a même le sentiment d'avoir été doublé :

«Sans aucun doute, nous serons heureux de la part de coopération fournie par le nouvel auxiliaire qui nous arrive; nous reconnaîtrons même que, parmi les collaborateurs de La Gazette des campagnes, il y a des écrivains que la France agricole aime depuis longtemps. Mais, puisque l'on nous amène sur un terrain où c'est notre devoir d'invoquer nos antécédents et surtout de redire notre programme politique dans ses rapports avec les intérêts agricoles, nous ne pouvons pas reculer ${ }^{67}$.»

Ce qui le gêne d'emblée, en effet, c'est moins l'« esprit de parti » que l'orientation politique que risque de prendre un parti agricole animé par Louis Hervé.

64. Barral (J.-A.), « Chronique agricole (première quinzaine d'octobre 1868) », op. cit., p. 159.

65. Barral (J.-A.), « Chronique agricole (première quinzaine d'octobre 1868) », op. cit., p. 158.

66. Hervé (L.), «La presse agricole aux élections », La Gazette des campagnes, 22 mai 1869, p. 162.

67. Lecouteux (E.), «L'agriculture et les prochaines élections générales », op. cit., p. 481-482. 
Les élections de 1869 doivent certes être selon lui « une puissante manifestation de l'esprit rural », mais non au sens où Louis Hervé et certains de ses amis pourraient le penser, non « cet ancien esprit rural qui ne connaissait que le principe d'autorité et qui reculait devant toute responsabilité, mais [...] cet esprit rural nouveau qui, plus que toute autre force sociale, peut concilier enfin l'ordre avec la liberté, c'est-à-dire le passé avec l'avenir ». Son inquiétude, on le voit, porte sur le caractère réactionnaire du parti de Louis Hervé, qui semble nostalgique de l'Ancien Régime et donc peu enclin à défendre les « principes politiques de 1789 », que revendique au contraire Lecouteux ${ }^{68}$.

Or, quelques semaines plus tard, les réactions suscitées par l'initiative de Louis Hervé confirment ses craintes. La véhémence de Louis Hervé contre la presse parisienne, l'industrie et le commerce, la finance et la civilisation urbaine en général, ses relations avec les milieux catholiques et monarchistes ont provoqué la colère d'une partie de l'opinion publique, des bonapartistes aux radicaux : "La riposte ne se fait pas attendre, note ainsi Lecouteux dès le mois de novembre, les passions entrent en scène, et bientôt s'élèvent dans le camp des adversaires du nouveau parti, de bruyantes clameurs où s'entrechoquent les accusations de parti féodal, de vieux partis coalisés, de ligue de la grande propriété, de hauts barons de la terre, d'obscurantisme, de cléricalisme, etc., etc. ${ }^{69}$. » Ces grands propriétaires terriens entrant en politique au nom des populations rurales ont en effet fait ressurgir le spectre de l'Ancien Régime, que leurs adversaires ont beau jeu d'agiter devant un électorat paysan globalement attaché aux acquis de $1789^{70}$.

Le modèle de S. M. Lipset et S. Rokkan paraît sur ce point confirmé. La défense des intérêts ruraux par Louis Hervé se confond avec celle du catholicisme et des intérêts des possédants, dans une superposition des différents clivages (campagne/ville, Église/État, capital/travail et même périphérie/centre) qui la conduit tôt ou tard à intégrer le programme des partis conservateurs ${ }^{71}$. La remarque de J.-M. De Waele et D.-L. Seiler trouve également écho dans la façon

68. Ibid., p. 483. Les mêmes motifs ont sans doute compté dans la défection de Lavergne, proche de l'Union libérale.

69. Lecouteux (E.), « Le parti de l'agriculture », op. cit., p. 641.

70. C'est ce que semblent indiquer les travaux consacrés à la politisation des électeurs ruraux au XIX siècle. Le soutien massif apporté par les paysans au régime bonapartiste est généralement interprété comme le refus de tout retour à l'Ancien Régime, même s'il repose sur des motifs très différents en fonction des régions. Dans certaines circonscriptions, les anciens notables réactionnaires se sont certes ralliés au bonapartisme, ce qui a pu faire croire au maintien de leur domination sur les paysans, qui les auraient suivi dans leur vote. Cf. Vigier (P.), « Le bonapartisme et le monde rural », in Hammer (K.), Hartmann (P. C.), dir., Le bonapartisme: phénomène historique et mythe politique, Munich, Artemis Verlag, 1977. Mais il est plus probable que ces notables aient plutôt suivi l'impulsion des paysans. Comme le souligne après d'autres Frédéric Bluche, ces derniers se montrent globalement attachés au principe égalitaire proclamé par la Révolution : « égalité, ordre, autorité : dès 1851, le bonapartisme avait tous les atouts en main ». Cf. Bluche (F.), Le bonapartisme: aux origines de la droite autoritaire (1800-1850), Paris, Nouvelles Éditions latines, 1980, p. 328.

71. Lipset (S. M.), Rokkan (S.), Structures de clivages, systèmes de partis et alignement des électeurs..., op. cit. 
dont la mémoire politique est instrumentalisée à l'occasion des élections : le passé féodal, fantasmé, constitue alors une ressource politique encore très efficace pour monter l'électorat paysan contre des élites rurales qui n'ont pourtant plus grand-chose des seigneurs médiévaux... L'indépendance des populations rurales à l'égard des élites agricoles, qui, ailleurs, sous-tend l'émergence d'un parti agraire autonome, se heurte ici à la configuration de la scène politique, qui tend à rabattre à droite la défense des intérêts ruraux. Il en résulte un échiquier politique paradoxal, où les porte-parole autoproclamés de l'agriculture semblent incapables de capter leur électorat de prédilection.

\section{Groupe de pression vs parti politique : 3-0}

Une telle conclusion a cependant quelque chose de téléologique. Elle repose en effet sur le présupposé selon lequel seul un Louis Hervé, réactionnaire et catholique, pouvait lancer un parti de l'agriculture - et donc échouer à donner à son entreprise l'autonomie politique qu'il souhaitait, alors même que les animateurs du mouvement rural sont loin de partager tous sa position politique. Elle s'appuie en outre sur une description en partie erronée de l'échiquier politique français. Les partis conservateurs n'étaient en réalité pas seuls à défendre les intérêts des ruraux. À une époque où l'électorat paysan est prépondérant, tous les partis se montrent en effet soucieux des questions agricoles et les mettent en avant dans leur programme ${ }^{72}$. La question doit donc être reformulée. Il ne s'agit pas de s'interroger sur la raison pour laquelle les partis politiques français préferent l'abstraction des grandes idées aux enjeux matériels - les programmes en sont pleins en ce milieu du XIX siècle. Il ne s'agit pas non plus de se demander pourquoi la défense de l'agriculture s'est trouvée prise en charge par les partis de droite plutôt que de s'autonomiser en un parti agraire - des bonapartistes aux socialistes en passant par les républicains, tous affirment alors être les mieux placés pour satisfaire les besoins des campagnes, qu'ils définissent diversement, en fonction de leurs projets politiques. Il s'agit bien plutôt de se demander pourquoi les animateurs du mouvement rural euxmêmes ont préféré le groupe de pression à l'aventure partisane qui leur était proposée par Louis Hervé.

Trois raisons apparaissent en filigrane des justifications avancées pour expliquer leur désaffection. La première est suggérée par la chronologie. Après avoir indiqué leur soutien à Louis Hervé, qui s'est déclaré tout début septembre, Barral comme Lecouteux prennent leurs distances début novembre, au moment où l'initiative de Louis Hervé commence à être commentée dans la presse politique. Le parti de l'agriculture apparait en effet comme un concurrent suffisamment sérieux pour être vigoureusement attaqué, notamment par les organes du

72. Barral (P.), Les agrariens français de Méline à Pisani, op. cit.; Cornu (P.), Mayaud (J.-L.), dir., Au nom de la terre..., op. cit.; Gaboriaux (C.), La République..., op. cit. 
gouvernement. Certains sont presque menaçants, comme le très bonapartiste La Patrie ${ }^{73}$, à qui Lecouteux se sent obligé d'adresser une longue lettre de dénégation, publiée début novembre dans Le Journal d'agriculture pratique. Or les activités du mouvement rural avaient pourtant jusqu'ici suscité la bienveillance du pouvoir... L'épisode révèle, s’il était besoin, les conditions de la générosité gouvernementale, qui lui est acquise tant qu'il reste apolitique. L'aventure partisane vaut-elle de risquer le soutien des autorités? Rien n'est moins sûr aux yeux des Lecouteux ou Barral.

Le risque est grand en effet de tout perdre. Quoiqu'elles ne cessent de répéter combien leurs intérêts se confondent avec ceux du plus grand nombre, les élites rurales ont conscience qu'il n'est pas facile de convaincre les électeurs paysans de voter pour elles. Les rares candidatures exclusivement agricoles ont ainsi régulièrement échoué aux élections générales. Or les leçons qu’en tirent nos publicistes suggèrent qu'ils ne sont pas prêts à se lancer à la conquête électorale des campagnes. Comme Lecouteux et Barral, Louis Hervé se contente au fil des pages de déplorer l'apathie des populations rurales, qui n'ont selon lui ni «le dévouement au devoir », ni « le sentiment de leurs droits », ni « la conscience de leurs forces », ni « l'intelligence de leurs véritables intérêts ${ }^{74}$ ». L'idée ne les avait pas effleurés que l'électorat rural pouvait à bon droit penser qu'il n'était ni dans son « devoir ", ni dans ses « droits ", ni même dans ses "véritables intérêts » de voter pour ces gros exploitants ou autres illustres agronomes. Dans la plupart des régions, on l'a dit, le bonapartisme majoritaire chez les paysans relevait plutôt d'un mouvement d'émancipation à l'égard de ces anciens notables... qui pouvaient en outre craindre les représailles du gouvernement partout où ils se présenteraient contre les candidats officiels ${ }^{75}$. La paysannerie était ainsi au centre de toutes les sollicitudes, celles des élites rurales qui s'autoproclamaient porte-parole de leurs intérêts pour assurer leurs positions de conseil auprès du gouvernement, celles des élus qui cherchaient à satisfaire leurs besoins en vue de se maintenir au pouvoir. On peut à cet égard imaginer que les électeurs ruraux y trouvaient leur compte et qu'ils étaient de ce fait peu encouragés à s'organiser pour formuler et faire valoir des aspirations que leur seul vote les assurait de voir satisfaites ${ }^{76}$.

Pour se lancer à la conquête de l'électorat, il aurait de toute façon fallu que les élites rurales fussent à même de parler d'une seule voix. Or, et c'est la troisième raison qui se cache sans doute derrière leur refus de l' « esprit de parti »,

73. Lannau-Rolland (A.), La Patrie, 24 octobre 1868, p. 1.

74. Hervé (L.), « La politique rurale », La Gazette des campagnes, 31 juillet 1869.

75. Lagoueyte (P.), Candidature officielle et pratiques électorales sous le Second Empire (1852-1870), thèse de doctorat sous la direction de Maurice Agulhon, Paris, Université Panthéon-Sorbonne, 1991.

76. «On ne mesure pas assez, je crois, note ainsi Philippe Vigier, l'importance des mesures pratiques prises par le régime impérial pour améliorer le sort des ruraux». Cf. Vigier (P.), « Le bonapartisme et le monde rural », art. cit., p. 18. Cf. aussi Agulhon (M.), « Les paysans dans la vie politique », in Duby (G.), Wallon (A.), dir., Histoire de la France rurale, Paris, Seuil, 1976, p. 329-355. 
elles formaient un groupe socialement hétérogène, formé de vieilles familles terriennes et de bourgeois ayant accédé à la propriété et/ou passés par les écoles - Polytechnique pour Barral, Grignon pour Lecouteux - et politiquement divisé : «naufragés » et "vaincus » des révolutions du siècle, ainsi que les appelle Lecouteux ${ }^{77}$, ces notables anciens et nouveaux n'ont finalement jamais partagé que le souci de défendre les intérêts agricoles... sans être d'ailleurs toujours d'accord sur ce qu'ils recouvraient. Si leurs différends les empêchent de fonder un parti, ils ont peu d'incidence sur l'efficacité de leur groupe de pression, au sein duquel ils sont tantôt occultés par le caractère purement agricole des débats, tantôt formulés dans les termes pacifiés de la discussion scientifique et technique. À l'heure où l'électorat était majoritairement rural, chacun avait ainsi intérêt au statu quo: le gouvernement, qui canalisait les ambitions des élites agricoles en leur assurant une position de conseil dépolitisé ; les électeurs ruraux qui pouvaient se tourner tantôt vers les élites agricoles, tantôt vers leurs élus pour faire entendre leurs revendications; les élites agricoles surtout qui, conscientes des aléas inhérents à la formation partisane, conservaient grâce au groupe de pression un accès privilégié au gouvernement quel que fût le résultat des élections.

À l'issue de notre enquête, nous pouvons donc affirmer d'abord que les deux hypothèses évoquées pour commencer - celle de la culture universaliste et celle des clivages - quoique d'horizons très différents, ne sont pas incompatibles. Le souci de la généralité est en effet indissociable de la configuration politique du moment. D'une part, la démocratie autoritaire attise la hantise des partis - mot d'ordre ancien (déjà employé pour justifier la montée de l'absolutisme puis le geste révolutionnaire), reformulé ici au profit du pouvoir de l'" hommepeuple $^{78} »$, qui s'efforce de contrôler l'échiquier politique. D'autre part, bien que les élites agricoles gardent une certaine distance avec le régime impérial, elles partagent jusqu'à un certain point sa culture illibérale. La hantise des partis constitue ainsi pour elles la formule qui exprime le plus fidèlement à leurs yeux la réalité des clivages auxquels elles sont confrontées et la justification qui leur permet d'y faire face dans les meilleures conditions.

À cet égard, il nous faut aussi amender en partie le modèle des clivages qui ne rend compte que partiellement de l'échec du parti de l'agriculture. Il permet certes de souligner le caractère paradoxal du cas français : son passé féodal, les tensions entre Église et État, le poids du catholicisme, sont en effet des facteurs qui, en France comme dans le reste de l'Europe, freinent l'émergence de partis agraires; mais si l'on en croit S. M. Lipset et S. Rokkan, l'indépendance relative d'un électorat paysan ultra-majoritaire aurait dû à l'inverse jouer en faveur

77. Lecouteux (E.), « Le parti de l'agriculture », op. cit., p. 643.

78. Cf. les développements de Pierre Rosanvallon à propos de la « démocratie illibérale » dans La démocratie inachevée : histoire de la souveraineté du peuple en France, Paris, Gallimard, 2000, p. 197 et s. 
de l'autonomisation de la défense des intérêts ruraux. On pourrait se contenter d'en conclure que les clivages liés à la révolution nationale (État/Église et centre/périphérie) pèsent ici plus lourd et rendent difficile l'émergence d'un parti essentiellement axé sur la défense des intérêts des campagnes. Mais il faut encore expliquer pourquoi ils ne conduisent pas cette dernière à être exclusivement prise en charge par le programme des partis conservateurs.

Ce que montre notre étude, c'est la nécessité de tenir compte de la reconfiguration politique particulière que constitue le Second Empire, à savoir une démocratisation de la vie politique sous l'égide d'un régime autoritaire. Le suffrage universel masculin, instauré dans un pays encore majoritairement rural, contribue à l'émancipation des populations rurales et fait de leurs intérêts un enjeu électoral majeur. Mais l'opportunité qu'il représente pour la constitution d'un parti agraire autonome est remise en question par la répression politique et la concentration des ressources étatiques dans les mains des bonapartistes. Ces deux contraintes se nourrissent et participent tout à la fois des différentes formes d'illibéralisme qui imprègnent une partie importante de la classe politique (dans une version politique du côté des bonapartistes, plus technocratique du côté des élites agricoles) ${ }^{79}$. Comprises et exprimées dans les termes de la hantise des partis, elles incitent les élites agricoles à préférer la voie corporatiste à la forme partisane, inaugurant un mode de gestion des intérêts agricoles dont le pays reste tributaire jusqu'à nos jours, alors même que les populations rurales ont perdu la force du nombre.

Chloé Gaboriaux est maîtresse de conférences en science politique à Sciences Po Lyon / Triangle UMR 5206 et directrice de la revue Mots. Les langages du politique. Ses recherches sur l'avènement de la République en France la conduisent à travailler les questions de la politisation et du rapport État/société civile, notamment à partir du rôle assigné aux corps intermédiaires. Elle est l'auteure de La République en quête de citoyens: les républicains français face au bonapartisme rural 18481880 (Presses de Sciences Po, 2010) et a codirigé avec Arnault Skornicki Vers une histoire sociale des idées politiques (Presses du Septentrion, 2017). Elle vient de publier dans Genèses "Une construction sociale de I'utilité publique. Associations et fondations devant le Conseil d'État (1870-1914) " (n¹09, 2017).

chloe.gaboriaux@sciencespo-lyon.fr

79. À propos des rapports entre discours et pratiques, cf. Gaboriaux (C.), « Lire et dire la société : de l’histoire conceptuelle du politique à l'histoire sociale des idées politiques », in Gaboriaux (C.), Skornicki (A.), dir., Vers une histoire sociale des idées politiques, Lille, Presses universitaires du Septentrion, 2017, p. 265-278. 
The weakness of agrarian parties in France: The case of the "party of agriculture" under the Second Empire

Little known to researchers, the debates raised in the late 1860s by the vain efforts of a publicist to launch a "party of agriculture" nevertheless provide many lessons. Whereas parties were being structured in a way that led them, at the end of the nineteenth century, to become parties in the modern sense of the word, this case enables us to better understand the paradox of the absence of a true agricultural or peasant party in France-a country where rural people have long constituted an overwhelming electoral majority. Distrust for parties, often invoked, is not sufficient to account for the failure of the "party of agriculture" under the Second Empire. It is, above all, an effective argument for its opponents, in a conflict that rural voters finally resolved against their self-proclaimed spokespersons. Reluctant to mistake their interests for those of the agricultural elites, the peasantry was also attracted by the arguments of their adversaries, who did not hesitate to denounce them as former notables eager to re-establish feudal relations. In a France where power relations are forcefully determined by the social and political imagination left behind by the Revolution, the "party of agriculture" was doomed to failure, not in spite of, but because of the electoral predominance of rural people.

Keywords-political parties, rural populations, agrarianism, representation, nineteenthcentury France 